\title{
ZEROS OF DIFFERENTIALS ALONG ONE-FIBERED IDEALS
}

\author{
R. FEDDER, C. HUNEKE AND R. HÜBL
}

(Communicated by Louis J. Ratliff, Jr.)

\begin{abstract}
Let $(R, m)$ be a complete local domain containing the rationals. If $I \subseteq R$ is a one-fibered ideal then there is a constant $l$, depending only on $R$ and $I$, such that if $f \in m$ and $f \notin I^{n}$, then there exists a derivation $d$ such that $d(f) \notin I^{n+l}$.
\end{abstract}

M. Hochster and C. Huneke have recently introduced the notion of tight closure of ideals and submodules. They proved, under some mild conditions, that an isolated singularity in which every ideal generated by a system of parameters is tightly closed is a rational singularity in characteristic 0 ([HH, (4.2), (4.1)]). It is conjectured that the converse of this statement holds true as well. Using an idea employed by Fedder in $[F]$ it would be a key point in the proof of the converse to prove the following statement:

Let $(R, m)$ be a complete local domain containing a field, let $K \subseteq R$ be a coefficient field, and assume that $K$ is perfect. Furthermore let $I \subseteq R$ be a one-fibered ideal (see Definition 1.1 below). Then there exists an $l=l(R, I)$, depending on $R$ and $I$ only, satisfying the following condition:

If $f \in R$ with $\delta(f) \in I^{n}$ for all derivations $\delta: R \rightarrow R$ then there exists a $g \in R$ such that $f+g \in I^{n-l}$ and $d(g)=0$, where

$$
d: R \rightarrow \widetilde{\Omega}_{R / K}^{1}
$$

is the universally finite derivation of $R / K$ ([KD, $\S 11])$.

In this note we show that this statement holds true if $\operatorname{char}(K)=0$, in which case $g=0$ if $f \in m$. In characteristic $p$, in the graded case, Fedder has some partial results on this question (cf. [F]).

In dimension 2, all rational singularities possess one-fibered ideals, so in the context of trying to show that system of parameters are tightly closed for isolated singularities which are rational, it is reasonable to assume the existence of such ideals. Not all local rings have such ideals, however (cf. [C]). All rings in this paper are commutative and noetherian.

Received by the editors January 30, 1989 and, in revised form April 11, 1989.

1980 Mathematics Subject Classification (1985 Revision). Primary 13H10.

Key words and phrases. One-fibered ideal, differentials.

The second author was partially supported by the National Science Foundation. 
Let $(R, m)$ be a local domain and let $I \subseteq R$ be an $m$-primary ideal. Denote by $\mathscr{R}(I)=R[I t]$ the corresponding Rees-ring, and by $\overline{\mathscr{R}(I)}$ its normalization.

Definition $1.1([\mathrm{~S}])$. The ideal $I$ is called one-fibered if $\operatorname{rad}(I \overline{\mathscr{R}}(I))$ is a prime ideal, i.e., if $I$ has only one associated Rees-valuation. (See [Mc, Ch.XI] for the definition of Rees valuations.)

Remark 1.2. One can characterize one-fibered ideals in several ways. Samuel defined the following function: $v_{I}(x)=$ largest integer $n$ such that $x \in$ $I^{n}$. This function is the "order" function of $R$ along $I$. Define $\bar{v}_{I}(x)=$ $\lim _{n \rightarrow \infty} v_{I}\left(x^{n}\right) / n$. Samuel [Sa] showed this limit exists while Rees [R2] proved that $\bar{v}_{I}(x)$ is a rational number and $\bar{v}_{I}(x) \geq n$ iff $x \in \overline{I^{n}}$, where for any ideal $J \subseteq R$, the integral closure of $J, \bar{J}$, is the set of elements $x \in R$ which satisfy an equation

$$
x^{n}+a_{1} x^{n-1}+\cdots+a_{n}=0,
$$

where $a_{i} \in J^{i}, 1 \leq i \leq n$ (cf. [P]).

It is known (cf. [S]) that $I$ is one-fibered iff $\bar{v}_{I}$ is a discrete valuation.

Suppose that $R$ is Noetherian, local, analytically unramified and that $\overline{I^{n}}$ are valuation ideals for some rank 1 discrete valuation $v$ for all $n$. Then $I$ is necessarily one-fibered. In fact, we will show that $\bar{v}_{I}(x)=v(x) / v(I)$, which implies that $\bar{v}_{\underline{I}}$ is a valuation. Let $v(x)=k, n=v(I)$. Then $v\left(x^{n l}\right)=v(I) l k$, so that $x^{n l} \in \overline{I^{l k}} \backslash \overline{I^{l k+1}}$. As $R$ is analytically unramified, there is a constant $c$ such that $\overline{I^{n}} \subseteq I^{n-c}$. Hence, $x^{n l} \notin I^{l k+1}$, but $x^{n l} \in I^{l k-c}$, for all $l$. Thus $(l k-c) / l n \leq v_{I}\left(x^{n l}\right) / n l<(l k+1) / l n$. Taking limits as $l \rightarrow \infty$ gives that $v(x) / v(I)=k / n=\bar{v}_{I}(x)$, as required.

Lemma 1.3. Let $(R, m)$ be an analytically unramified one-dimensional local domain with integral closure $S$. Then the following conditions are equivalent:

(i) $S$ is local.

(ii) There exists a 1-fibered ideal $I \subseteq R$.

(iii) Every nonzero ideal $I \subseteq R$ is 1-fibered.

Proof. Clearly (iii) implies (ii). We will show first that (ii) implies (i). By [S, Prop., p. 4], $R$ is analytically irreducible. If $S$ was not local, it would have at least two maximal ideals, which forces $\widehat{R}$ to have at least two minimal primes. This contradiction shows that $S$ is local, which proves (i).

Next we show that (i) implies (iii). Since $S$ is local, $S$ is a rank 1 discrete valuation ring (DVR). Furthermore, if $I \subseteq R$, then $\overline{I^{n}}=I^{n} S \cap R$ since $S$ is the integral closure of $R$. Hence $\overline{I^{n}}$ are valuation ideals for the DVR $S$, and by Remark 1.2 above this implies that $I$ is one-fibered. 
A key point in the proof of the theorem mentioned above is the following result:

Theorem 1.4. Let $(R, m)$ be a d-dimensional analytically unramified domain, let $I \subseteq R$ be a one-fibered m-primary ideal, and suppose that $R$ has an infinite residue field $k=R / m$. Then there exists an $l=l(R, I)$ satisfying the following:

If $f \in m$ and $f \notin I^{n}$ then there exist $g_{2}, \ldots, g_{d} \in I$ such that $\left(f, g_{2}, \ldots, g_{d}\right) \supseteq I^{n+l}$.

Proof. Case 1. $d=1$. Note that the existence of a one-fibered ideal implies that $R$ is analytically irreducible ([S, Prop., p. 4]), i.e., that $\widehat{R}$, the $m$-adic completion of $R$, is a domain. By the faithful flatness of $\widehat{R} / R$ it obviously suffices to show that there exists an $l$ such that whenever $f \in m, f \notin I^{n}$ then $f \widehat{R} \supseteq I^{n+l} \widehat{R}$. Let $S=\overline{\widehat{R}}$ be the integral closure of $\widehat{R}$ in the quotient field $K$ of $\widehat{R}$. Then $S$ is a finite $\widehat{R}$-module since $\widehat{R}$ is Nagata, and $S$ is local. By the Artin-Rees Lemma there exists an $l_{1} \in \mathbf{N}$ such that $I^{n+l_{1}} S \cap \widehat{R}=I^{n}\left(\widehat{R} \cap I^{l_{1}} S\right) \subseteq$ $I^{n} \widehat{R}$ for all $n$. So from $f \notin I^{n}$ it follows $f \notin I^{n+l_{1}} S$. Since $S$ is a DVR we get $I S=x S$ for some $x \in S$, and furthermore $f S \supseteq I^{n+l_{1}} S$.

Denote by $\mathscr{L}_{S / \widehat{R}}$ the conductor from $S$ to $\widehat{R}$. Since $S$ is a finite $\widehat{R}$-module $\mathscr{L}_{S / \widehat{R}}$ is an $m$-primary ideal, and therefore there exists an $l_{2} \in \mathbf{N}$ such that $I^{l_{2}} \widehat{R} \subseteq \mathscr{L}_{S / \widehat{R}}$. Then for $l=l_{1}+l_{2}$ it holds:

$$
f \widehat{R} \supseteq f \mathscr{L}_{S / \widehat{R}} S \supseteq f I^{l_{2}} S \supseteq I^{l_{2}} I^{n+l_{1}} S=I^{n+l} S \supseteq I^{n+l} \widehat{R}
$$

as desired.

Case 2. $d$ arbitrary. Let $J=\left(x_{1}, \ldots, x_{d}\right)$ be a minimal reduction (see [NR]) of $I$. Then $J$ is a one-fibered ideal as well since $\overline{\mathscr{R}(J)}=\overline{\mathscr{R}(I)}$. Suppose we have shown the theorem for $J$, with an integer $l^{\prime}=l(R, J)$. Since $J$ is a reduction of $I$, there is an integer $t$ such that $I^{t+n}=J^{n} I^{t}$. If $f \notin I^{n}$, then $f \notin J^{n}$ and therefore there exist $g_{2}, \ldots, g_{d} \in J$ such that $\left(f, g_{2}, \ldots, g_{d}\right) \supseteq$ $J^{n+l^{\prime}}$. But $J^{n+l^{\prime}} \supseteq I^{n+l^{\prime}+t}=I^{n+l}$ which proves the theorem for $I$. Therefore we may assume $I=J$, i.e., $I$ is generated by $d$ elements. Denote by $S=$ $R\left[x_{2} / x_{1}, \ldots, x_{d} / x_{1}\right]$ the degree zero component of $\mathscr{R}(I)_{x_{1}}$, and let $T=\bar{S}$ be the integral closure of $S$. Furthermore, let $Y_{2}, \ldots, Y_{d}$ be indeterminates, denote by $u_{i}=x_{1} Y_{i}-x_{i} \in R\left[Y_{2}, \ldots, Y_{d}\right]$, and denote by $p$ the kernel of the $R$-epimorphism

$$
\varphi: R\left[Y_{2}, \ldots, Y_{d}\right] \rightarrow R\left[x_{2} / x_{1}, \ldots, x_{d} / x_{1}\right], \quad Y_{i} \mapsto x_{i} / x_{1} .
$$

Lemma 1.5. There exists a $k \in \mathbf{N}$ such that

$$
I^{k} p \subseteq\left(u_{2}, \ldots, u_{d}\right) .
$$

The proof of this lemma will be given after the conclusion of the proof of Theorem 1.4. 
Since $I$ is a one-fibered ideal $\operatorname{rad}(I T)=Q$ is a prime ideal of $T$. Denote by $P=Q \cap S$. By the valuative criterion of integral dependence we get

$$
I^{r} T_{Q} \cap R=\overline{I^{r}}
$$

Note that $\operatorname{dim}\left(S_{P}\right)=1=\operatorname{dim}\left(T_{Q}\right)$.

As $R$ is analytically unramified there exists an $l_{1}=l_{1}(R, I)$ such that $\overline{I^{n+l_{1}}} \subseteq$ $I^{n}$ for all $n \geq 0$ ([R1, (1.4)]), and therefore if $f \notin I^{n}$ we have that $f \notin$ $I^{n+l_{1}} S_{P}$. By a theorem of Rees ([R1, (1.5)]) $T$ is a finite $S$-module. Since $I$ is one-fibered, $T_{Q}=T_{P}\left(=T \otimes_{S} S_{P}\right)$, and therefore the integral closure of $S_{P}$, which is $T_{P}$, is local, and it is a finite $S_{P}$-module, implying that $I S_{P}$ is one-fibered by Lemma 1.3 and that $S_{P}$ is analytically unramified. Therefore we can apply the case $d=1$ to $I S_{P}$, and we get that there exists an $l_{2}=l_{2}(R, I)$ such that whenever $f \notin I^{n} S_{P}$ then $f S_{P} \supseteq I^{n+l_{2}} S_{P}$.

We get for $l_{3}=l_{1}+l_{2}$ that whenever $f \in R, f \notin I^{n}$ then $f S_{P} \supseteq I^{n+l_{3}} S_{P}$. Therefore there exists a $q \in S \backslash P$ such that

$$
f S_{q} \supseteq I^{n+l_{3}} S_{q},
$$

i.e., such that

$$
f\left(R\left[Y_{2}, \ldots, Y_{d}\right] / p\right)_{q} \supseteq I^{n+l_{3}}\left(R\left[Y_{2}, \ldots, Y_{d}\right] / p\right)_{q},
$$

implying that

$$
(f, p) R\left[Y_{2}, \ldots, Y_{d}\right]_{q(Y)} \supseteq I^{n+l_{3}} R\left[Y_{2}, \ldots, Y_{d}\right]_{q(Y)},
$$

where $q(Y)$ is a preimage of $q$ in $R\left[Y_{2}, \ldots, Y_{d}\right]$. Now let $k$ be as in Lemma 1.5 and let $l=l_{3}+k$. Then

$$
\left(f, u_{2}, \ldots, u_{d}\right) R\left[Y_{2}, \ldots, Y_{d}\right]_{q(Y)} \supseteq I^{n+l} R\left[Y_{2}, \ldots, Y_{d}\right]_{q(Y)}
$$

by the lemma, with $l$ depending on $R$ and $I$ only. Since $q(Y) \notin m(R) \times$ $\left[Y_{2}, \ldots, Y_{d}\right]$ and since $R / m$ is infinite there exist $\alpha_{2}, \ldots, \alpha_{d} \in R$ such that $q\left(\alpha_{2}, \ldots, \alpha_{d}\right) \notin m$, and therefore $\alpha_{2}, \ldots, \alpha_{d}$ induce an $R$-epimorphism

$$
\psi: R\left[Y_{2}, \ldots, Y_{d}\right]_{q(Y)} \rightarrow R,
$$

and with $g_{i}:=\psi\left(u_{i}\right) \quad(i=2, \ldots, d)$ we get $g_{i} \in I$, and, by the above,

$$
\begin{aligned}
\left(f, g_{2}, \ldots, g_{d}\right) & =\psi\left(\left(f, u_{2}, \ldots, u_{d}\right) R\left[Y_{2}, \ldots, Y_{d}\right]_{q(Y)}\right) \\
& \supseteq \psi\left(I^{n+l} R\left[Y_{2}, \ldots, Y_{d}\right]_{q(Y)}\right) \\
& =I^{n+l} .
\end{aligned}
$$

Proof of Lemma 1.5. The map

$$
\varphi: R\left[Y_{2}, \ldots, Y_{d}\right] \rightarrow R\left[x_{2} / x_{1}, \ldots, x_{d} / x_{1}\right], \quad Y_{i} \mapsto x_{i} / x_{1},
$$

arises from the homomorphism

$$
\phi: R\left[Z_{1}, \ldots, Z_{d}\right] \rightarrow R\left[x_{1} T, \ldots, x_{d} T\right], \quad Z_{i} \mapsto x_{i} T
$$


by localizing in $Z_{1}$, resp. $x_{1} T$, taking degree zero components and identifying $Z_{j} / Z_{1}$ with $Y_{j}$. Let $P$ be the kernel of $\phi$ and denote by $a_{i j}=Z_{i} x_{j}-x_{i} Z_{j} \in P$. Furthermore denote by $A=\left(a_{i j}\right)$ the homogeneous ideal generated by the elements $a_{i j}$. Denote by $\left(A_{Z_{1}}\right)_{0}$ the degree zero component of the homogeneous ideal $A R\left[Z_{1}, \ldots, Z_{d}\right]_{Z_{1}}$. Then $\left(A_{Z_{1}}\right)_{0}=\left(u_{2}, \ldots, u_{d}\right)$ since $x_{i} Y_{j}-x_{j} Y_{i}=$ $Y_{i}\left(x_{1} Y_{j}-x_{j}\right)-Y_{j}\left(x_{1} Y_{i}-x_{i}\right)$, and therefore it suffices to show

$$
I^{k} P \subseteq A \quad \text { for some } k \text {. }
$$

For this it obviously is enough to show that

$$
x_{i}^{n} P \subseteq A \quad \text { for some } n \in \mathbf{N} \quad(i=1, \ldots, d)
$$

or equivalently

$$
P R\left[Z_{1}, \ldots, Z_{d}\right]_{x_{i}}=A R\left[Z_{1}, \ldots, Z_{d}\right]_{x_{i}} \text {. }
$$

By symmetry we may assume $i=1$. Then $A_{x_{1}}$ is obviously generated by the elements $Z_{i}-x_{i} / x_{1} Z_{1}$, and therefore $R\left[Z_{1}, \ldots, Z_{d}\right]_{x_{1}} / A_{x_{1}} \cong R_{x_{1}}\left[Z_{1}\right]$, so that $\phi$ induces a homogeneous $R$-epimorphism

$$
R\left[Z_{1}, \ldots, Z_{d}\right]_{x_{1}} / A_{x_{1}} \cong R_{x_{1}}\left[Z_{1}\right] \rightarrow R\left[x_{1} T, \ldots, x_{d} T\right]_{x_{1}}=R_{x_{1}}[T]
$$

which therefore has to be an isomorphism, implying $P_{x_{1}}=A_{x_{1}}$ as desired.

Remark. We do not know whether the converse of Theorem 1.4 holds true. $J$. Sally showed us a proof of the converse if $R$ is one-dimensional.

Theorem 1.6. Let $(R, m)$ be a complete local domain containing $\mathbf{Q}$, and let $I \subseteq$ $R$ be a one-fibered m-primary ideal. Then there exists an $l=l(R, I)$ satisfying the following condition: If $f \in m, f \notin I^{n}$ then there exists a derivation $d \in$ $\operatorname{Der}(R)$ such that $d(f) \notin I^{n+l}$.

The following easy consequence of the Artin-Rees Lemma will play a crucial role in the proof of Theorem 1.6:

Lemma 1.7. Let $R$ be a domain, let $M$ be a finitely generated torsion-free $R$ module and let $I \subseteq R$ be an ideal of $R$. Then there exists an $l=l(M, I)$ such that, whenever $m \notin I^{n} M$, then there exists a $f \in \operatorname{Hom}_{R}(M, R)$ with $f(m) \notin I^{n+l}$.

Proof. Since $R$ is a domain there exists a free $R$-module $F \subseteq M$ such that $F_{r}=M_{r}$ for some $r \in R \backslash\{0\}$. Since $M$ is finitely generated we may assume that $r M \subseteq F$. Let $f_{1}, \ldots, f_{d}$ be a basis of $F$ and denote by $\delta_{1}, \ldots, \delta_{d} \in$ $\operatorname{Hom}_{R}(F, R)$ its dual basis. Then it clearly holds: If $x \notin I^{n} F$ then there exists an $i \in\{1, \ldots, d\}$ such that $\delta_{i}(x) \notin I^{n}$.

By the Artin-Rees Lemma there exists an $l=l(M, I)$ such that, for all $n \in \mathbf{N}$,

$$
I^{n+l} M \cap r M=I^{n}\left(r M \cap I^{l} M\right) \subseteq I^{n} r M \subseteq I^{n} F .
$$

Since $M$ is torsion-free, the map $\mu_{r}: M \rightarrow r M(m \mapsto r m)$ is an isomorphism, and therefore if $m \notin I^{n} M$ then $r m \notin I^{n} r M$, hence $r m \notin I^{n}\left(r M \cap I^{l} M\right)=$ $I^{n+l} M \cap r M$. Since $r m \in r M$ this implies $r m \notin I^{n+l} M$, hence in particular 
$r m \notin I^{n+l} F$. So by the above $\delta_{i}(r m) \notin I^{n+l}$ for some $i \in\{1, \ldots, d\}$, and therefore the $R$-homomorphism $f$ given by

$$
f: M \stackrel{\mu_{r}}{\longrightarrow} r M \hookrightarrow F \stackrel{\delta_{i}}{\longrightarrow} R
$$

will satisfy $f(m) \notin I^{n+l}$, proving the lemma.

Proof of Theorem 1.6. Denote by $L=Q(R)$ the quotient field of $R$, let $k \subseteq R$ be a coefficient field of $R$, and let $\widetilde{\Omega}_{R / k}^{1}$ be the universally finite differential module of $R / k$ (cf. [KD, $\S 11]$ ), and denote by $\widetilde{\Omega}_{R / k}^{*}$ the module $\widetilde{\Omega}_{R / k}^{1} /$ torsion, which is the image of $\widetilde{\Omega}_{R / k}^{1}$ by the canonical map

$$
\widetilde{\Omega}_{R / k}^{1} \rightarrow\left(\widetilde{\Omega}_{R / k}^{1}\right) \otimes_{R} L=D_{k}(L) .
$$

We have canonical isomorphisms

$$
\operatorname{Der}_{k}(R) \cong \operatorname{Hom}_{R}\left(\widetilde{\Omega}_{R / k}^{1}, R\right) \cong \operatorname{Hom}_{R}\left(\widetilde{\Omega}_{R / k}^{*}, R\right)
$$

and therefore in view of Lemma 1.7 it suffices to show that there exists an $l_{1}=$ $l_{1}(R, I)$ such that the following holds: If $r \in m, r \notin I^{n}$ then $d r \notin I^{n+l_{1}} \widetilde{\Omega}_{R / k}^{*}$, where by abuse of notation $d$ denotes the composition

$$
R \stackrel{d}{\longrightarrow} \widetilde{\Omega}_{R / k}^{1} \stackrel{\text { can }}{\longrightarrow} \widetilde{\Omega}_{R / k}^{*} .
$$

Let $t=\operatorname{dim}(R)$ and let $l_{1}=l_{1}(R, I)$ be as in Theorem 1.4; i.e., if $r \notin I^{n}$ then there exist $g_{2}, \ldots, g_{t} \in I$ such that $\left(r, g_{2}, \ldots, g_{t}\right) \supseteq I^{n+l_{1}}$.

Since $\left(r, g_{2}, \ldots, g_{t}\right)$ is an $m$-primary ideal the inclusion

$$
P=k\left[\left[r, g_{2}, \ldots, g_{t}\right]\right] \hookrightarrow R
$$

is a finite morphism. Note that $P$ is a power series algebra in $r, g_{2}, \ldots, g_{t}$. Let $K=Q(P)$ be the quotient field of $P$ and let $D_{k}(K)=\widetilde{\Omega}_{P / k}^{1} \otimes_{P} K$. Then $D_{k}(L)$ is the universal $L$-extension of $D_{k}(K)$ by [KD, (11.9) and (4.21)], and therefore

$$
D_{k}(L)=L \otimes_{K} D_{k}(K)
$$

since $L / K$ is finite and separable $([\mathrm{KD},(5.2)])$. Hence the canonical trace ([KD, App. F])

$$
\sigma_{L / K}: L \rightarrow K
$$

induces a well defined $D_{k}(K)$-linear map

$$
\tilde{\sigma}_{L / K}: D_{k}(L) \rightarrow D_{k}(K)
$$

which satisfies

$$
\tilde{\sigma}_{L / K}\left(\widetilde{\Omega}_{R / k}^{*}\right) \subseteq \widetilde{\Omega}_{P / k}^{1}=P d r \oplus P d g_{2} \oplus \cdots \oplus P d g_{t}
$$

as follows easily from [KW, (5.26)]. Hence we get

$$
\tilde{\sigma}_{L / K}(d r)=d r \cdot \operatorname{dim}_{K}(L) \notin\left(r, g_{2}, \ldots, g_{t}\right) \widetilde{\Omega}_{P / k}^{1}
$$


and therefore

$$
d r \notin\left(r, g_{2}, \ldots, g_{t}\right) \widetilde{\Omega}_{R / k}^{*}
$$

by the $D_{k}(K)$-linearity of $\tilde{\sigma}_{L / K}$, implying

$$
d r \notin I^{n+l_{1}} \widetilde{\Omega}_{R / k}^{*}
$$

as desired.

\section{REFERENCES}

[C] D. Cutkosky, On unique and almost unique factorization of complete ideals II, (to appear in Inventiones Math.).

[F] R. Fedder, Rational singularity implies F-injective type for graded Cohen-Macaulay rings of dimension 2, (in preparation).

[HH] M. Hochster and C. Huneke, Tight closure, Proceedings of MSRI Microprogram on Commutative Algebra, MSRI Publ. 15 (1989), 305-324.

[KD] E. Kunz, Kähler differentials, Vieweg, Braunschweig, Wiesbaden, 1986.

[KW] E. Kunz and R. Waldi, Regular differential forms, Contemporary Mathematics, Amer. Math. Soc., Providence, R.I., 1988.

[Mc] S. McAdam, Asymptotic prime divisors, Lecture Notes in Math. 1023, Springer, Berlin, 1983.

[NR] D. G. Northcott and D. Rees, Reductions of ideals in local rings, Proc. Camb. Phil. Soc. 50 (1954), 145-158.

[P] H. Prüfer, Untersuchungen über Teilbarkeitseigenschaften in Körpern, J. Reine Angew. Math. 168 (1932), 1-36.

[R1] D. Rees, A note on analytically unramified local rings, J. London Math. Soc. 36 (1961), 24-28.

[R2] __ Valuations associated with ideals II, J. London Math. Soc. 31 (1956), 221-228.

[S] J. Sally, One-fibered ideals, Proceedings of MSRI Microprogram on Commutative Algebra, MSRI Publ. 15 (1989), 437-442.

[Sa] P. Samuel, Some asymptotic properties of powers of ideals, Annals of Math. 56 (1952), 11-21.

Department of Mathematics, Purdue University, West Lafayette, Indiana 47907 